\title{
CERTAIN THEOREMS ON BILINEAR AND BILATERAL GENERATING FUNCTIONS
}

\author{
H. M. SRIVASTAVA ${ }^{1}$ and YEONG-NAN YEH ${ }^{2}$
}

(Received 11 March, 1999)

\begin{abstract}
It is observed (among other things) that a theorem on bilinear and bilateral generating functions, which was given recently in the predecessor of this Journal, does not hold true as stated and proved earlier. Several possible remedies and generalizations, which indeed are relevant to the present investigation of various other results on bilinear and bilateral generating functions, are also considered.
\end{abstract}

\section{Introduction}

Consider a two-variable generating function $F(x, t)$ which possesses a formal (not necessarily convergent for $t \neq 0$ ) power-series expansion in $t$ such that

$$
F(x, t)=\sum_{n=0}^{\infty} c_{n} f_{n}(x) t^{n}
$$

where each member of the generated set $\left\{f_{n}(x)\right\}_{n=0}^{\infty}$ is independent of $t$, and the coefficient set $\left\{c_{n}\right\}_{n=0}^{\infty}$ may contain the parameters of the set $\left\{f_{n}(x)\right\}_{n=0}^{\infty}$, but is independent of $x$ and $t$. Also let $(\lambda)_{n}$ denote the Pochhammer symbol defined by

$$
(\lambda)_{n}:=\frac{\Gamma(\lambda+n)}{\Gamma(\lambda)}= \begin{cases}1, & n=0, \lambda \neq 0, \\ \lambda(\lambda+1) \cdots(\lambda+n-1), & n \in \mathbb{N}:=\{1,2,3, \ldots\} .\end{cases}
$$

In a recent paper published in the predecessor of this Journal, Mohammad [6] claimed to have proved an obvious special case of the assertion (6) of the following theorem

\footnotetext{
${ }^{1}$ Department of Mathematics and Statistics, University of Victoria, Victoria, British Columbia V8W 3P4, Canada; e-mail: hmsri@uvvm.uvic.ca.

${ }^{2}$ Institute of Mathematics, Academia Sinica, Taipei 11529, Taiwan, Republic of China; e-mail: mayeh@ccvax.sinica.edu.tw.

(C) Australian Mathematical Society 2002, Serial-fee code 1446-1811/02
} 
on bilinear and bilateral generating functions (cf. [6, p. 261, Theorem 1]) when

$$
r=3, \quad m_{j}=1(j=1,2,3) \text { and } c_{n}=1\left(n \in \mathbb{N}_{0}:=\mathbb{N} \cup\{0\}\right)
$$

THEOREM 1. For bounded complex coefficients $\Lambda\left(k_{1}, \ldots, k_{r}\right)\left(k_{j} \in \mathbb{N}_{0} ; j=1\right.$, $\ldots, r)$, let

$$
\Phi\left(z_{1}, \ldots, z_{r}\right):=\sum_{k_{1}, \ldots, k_{r}=0}^{\infty} \Lambda\left(k_{1}, \ldots, k_{r}\right) z_{1}^{k_{1}} \cdots z_{r}^{k_{r}}
$$

and

$$
\Phi^{*}\left(\lambda, \mu ; z_{1}, \ldots, z_{r}\right):=\sum_{k_{1}, \ldots, k_{r}=0}^{\infty} \frac{(\lambda)_{M}}{(\mu)_{M}} \Lambda\left(k_{1}, \ldots, k_{r}\right) z_{1}^{k_{1}} \cdots z_{r}^{k_{r}},
$$

where, for convenience,

$$
M:=m_{1} k_{1}+\cdots+m_{r} k_{r} \quad\left(m_{j} \in \mathbb{R}_{+} ; j=1, \ldots, r\right),
$$

the parameters $\lambda$ and $\mu$ (and the variables $\left|z_{1}\right|, \ldots,\left|z_{r}\right|$ ) being so constrained that the multiple series in (3) and (4) converge absolutely. Also let the generating function $F(x, t)$ defined by (1) be such that $F(x,-t u /(1-u))$ remains uniformly convergent for $u \in(0,1)$.

Then

$$
\begin{aligned}
& \sum_{n=0}^{\infty} \frac{(\lambda)_{n}}{(\lambda-\mu+1)_{n}} c_{n} \Phi^{*}\left(\lambda+n, \mu ; z_{1}, \ldots, z_{r}\right) f_{n}(x) t^{n} \\
& \quad=\frac{\Gamma(\mu)}{\Gamma(\lambda) \Gamma(\mu-\lambda)} \int_{0}^{1} \frac{u^{\lambda-1} \Phi\left(z_{1} u^{m_{1}}, \ldots, z_{r} u^{m_{r}}\right)}{(1-u)^{\lambda-\mu+1}} F\left(x,-\frac{t u}{1-u}\right) d u
\end{aligned}
$$

and

$$
\begin{aligned}
& \sum_{n=0}^{\infty} \frac{(1-\mu)_{n}}{(\lambda-\mu+1)_{n}} c_{n} \Phi^{*}\left(\lambda, \mu-n ; z_{1}, \ldots, z_{r}\right) f_{n}(x) t^{n} \\
& \quad=\frac{\Gamma(\mu)}{\Gamma(\lambda) \Gamma(\mu-\lambda)} \int_{0}^{1} \frac{u^{\lambda-1} \Phi\left(z_{1} u^{m_{1}}, \ldots, z_{r} u^{m_{r}}\right)}{(1-u)^{\lambda-\mu+1}} F\left(x, \frac{t}{1-u}\right) d u,
\end{aligned}
$$

provided that $\operatorname{Re}(\mu)>\operatorname{Re}(\lambda)>0$.

It should be remarked in passing that several other special cases of the assertions (6) as well as (7) of Theorem 1 (with $m_{j}=1, j=1, \ldots, r$ and $c_{n}=1, n \in \mathbb{N}_{0}$ ), associated with Lauricella's hypergeometric hypergeometric functions $F_{A}^{(r)}, F_{B}^{(r)}, F_{C}^{(r)}$, and $F_{D}^{(r)}$ of $r$ variables ( $c f$. [4, p. 113]; see also Srivastava and Karlsson [10, p. 33]), were given earlier by Mathur [5]. 


\section{Validity of Theorem 1}

In their derivation of the aforementioned special cases of Theorem 1, both Mohammad [6, p. 263] and Mathur [5, p. 222] made use of the familiar Eulerian (Betafunction) integral [3, p. 9 et seq.]:

$$
\int_{0}^{1} u^{\alpha-1}(1-u)^{\beta-1} d u=B(\alpha, \beta)=\frac{\Gamma(\alpha) \Gamma(\beta)}{\Gamma(\alpha+\beta)}, \quad \operatorname{Re}(\alpha)>0 ; \operatorname{Re}(\beta)>0,
$$

in conjunction with the power-series expansion (1). If, for convenience, we denote the right-hand side of the assertion (6) of Theorem 1 by $\Omega\left(z_{1}, \ldots, z_{r} ; t\right)$, and apply (1) as well as the definition (3), we readily find that

$$
\begin{aligned}
\Omega\left(z_{1}, \cdots, z_{r} ; t\right)= & \frac{\Gamma(\mu)}{\Gamma(\lambda) \Gamma(\mu-\lambda)} \sum_{n, k_{1}, \ldots, k_{r}=0}^{\infty} c_{n} \Lambda\left(k_{1}, \ldots, k_{r}\right) f_{n}(x) \\
& \times z_{1}^{k_{1}} \cdots z_{r}^{k_{r}}(-t)^{n} \int_{0}^{1} u^{\lambda+n+M-1}(1-u)^{\mu-\lambda-n-1} d u,
\end{aligned}
$$

provided that the inversion of the order of summation and integration is permissible under the hypotheses of Theorem 1, $M$ being given (as before) by (5).

Now, in order to evaluate the integral in (9) by means of the Eulerian integral (8) with $\alpha$ and $\beta$ replaced by $\lambda+n+M$ and $\mu-\lambda-n$, respectively, these parameters must be constrained by $\operatorname{Re}(\mu+M)>\operatorname{Re}(\lambda+n+M)>0$, that is, by

$$
\operatorname{Re}(\mu)>\operatorname{Re}(\lambda+n)>0, \quad n \in \mathbb{N}_{0} .
$$

The first inequality in (10), viz. $\operatorname{Re}(\mu)>\operatorname{Re}(\lambda+n), n \in \mathbb{N}_{0}$, cannot be satisfied for all bounded complex parameters $\lambda$ and $\mu$ as long as $n$ is unbounded above.

A similar problem arises in the derivation of the assertion (7) of Theorem 1. Indeed, if $\omega\left(z_{1}, \ldots, z_{r} ; t\right)$ denotes the right-hand side of (7), it is easily observed from (1) and (3) that

$$
\begin{aligned}
\omega\left(z_{1}, \cdots, z_{r} ; t\right)= & \frac{\Gamma(\mu)}{\Gamma(\lambda) \Gamma(\mu-\lambda)} \sum_{n, k_{1}, \ldots, k_{r}=0}^{\infty} c_{n} \Lambda\left(k_{1}, \ldots, k_{r}\right) f_{n}(x) \\
& \times z_{1}^{k_{1}} \cdots z_{r}^{k_{r}} t^{n} \int_{0}^{1} u^{\lambda+M-1}(1-u)^{\mu-\lambda-n-1} d u,
\end{aligned}
$$

in which the integral obviously diverges, since one of the convergence conditions ( $c f$. (10)): $\operatorname{Re}(\mu-n)>\operatorname{Re}(\lambda), n \in \mathbb{N}_{0}$, cannot be satisfied for all bounded complex parameters $\lambda$ and $\mu$ as long as $n$ is unbounded above.

Thus the derivation of Theorem 1, even in the aforementioned special cases considered by Mohammad [6] and Mathur [5], cannot be validated. And, since the integrals 
occurring in the assertions (6) and (7) are divergent, Theorem 1 does not hold true as stated and proved so far.

We choose to remark here that yet another special case of the evidently invalid assertion (6) of Theorem 1 when $m_{j}=1, j=1, \ldots, r$, happens to be one of the three main results derived in a forthcoming paper: "A certain class of bilateral generating functions involving generalized polynomials", by M. A. Pathan and M. G. Bin-Saad, which is scheduled to appear in The ANZIAM Journal.

\section{A set of possible remedies for Theorem 1}

One of several possibilities for correcting Theorem 1 would involve the use of Pochhammer's extension of the Eulerian integral (8) in the form ( $c f$. Whittaker and Watson [12, p. 256, Section 12.43]; see also Srivastava and Karlsson [10, p. 280]):

$$
\int_{P}^{(1+, 0+, 1-, 0-)}(-\zeta)^{\alpha-1}(\zeta-1)^{\beta-1} d \zeta=-\frac{4 \pi^{2}}{\Gamma(1-\alpha) \Gamma(1-\beta) \Gamma(\alpha+\beta)},
$$

where the path of integration is Pochhammer's double-loop which starts from a point $P$ on the real axis (of the complex $\zeta$-plane) between 0 and 1 , encircles the point 1 in the positive (counter-clockwise) direction and returns to $P$, then encircles the origin in the positive direction and returns to $P$, and so on. Denoting such a contour of integration simply as in $\int_{P}^{[0 ; 1]}$, we obtain the following corrected version of Theorem 1 .

THEOREM 2. With definitions and notation as in Theorem 1,

$$
\begin{aligned}
& \sum_{n=0}^{\infty} \frac{(\lambda)_{n}}{(\lambda-\mu+1)_{n}} c_{n} \Phi^{*}\left(\lambda+n, \mu ; z_{1}, \ldots, z_{r}\right) f_{n}(x) t^{n} \\
& =-\frac{\Gamma(1-\lambda) \Gamma(\lambda-\mu+1) \Gamma(\mu)}{4 \pi^{2}} \\
& \times \int_{P}^{[0 ; 1]}(-\zeta)^{\lambda-1}(\zeta-1)^{\mu-\lambda-1} \Phi\left(z_{1} \zeta^{m_{1}}, \ldots, z_{r} \zeta^{m_{r}}\right) F\left(x, \frac{\zeta t}{\zeta-1}\right) d \zeta
\end{aligned}
$$

and

$$
\begin{aligned}
\sum_{n=0}^{\infty} \frac{(1-\mu)_{n}}{(\lambda-\mu+1)_{n}} c_{n} \Phi^{*}\left(\lambda, \mu-n ; z_{1}, \ldots, z_{r}\right) f_{n}(x) t^{n} \\
=-\frac{\Gamma(1-\lambda) \Gamma(\lambda-\mu+1) \Gamma(\mu)}{4 \pi^{2}} \\
\quad \times \int_{P}^{[0: 11}(-\zeta)^{\lambda-1}(\zeta-1)^{\mu-\lambda-1} \Phi\left(z_{1} \zeta^{m_{1}}, \ldots, z_{r} \zeta^{m_{r}}\right) F\left(x, \frac{-t}{\zeta-1}\right) d \zeta,
\end{aligned}
$$

provided that $\lambda, \mu \in \mathbb{C} \backslash \mathbb{Z},(\mathbb{Z}:=\{0, \pm 1, \pm 2, \ldots\})$. 
Another useful extension of the Eulerian integral (8) involves a single-loop contour integral given by (see, for example, Erdélyi et al. [3, p. 15, Equation 1.6(8)])

$$
B(\alpha, \beta)=\frac{1}{2} \operatorname{csch}(\beta \pi i) \int_{0}^{(1+)} \zeta^{\alpha-1}(\zeta-1)^{\beta-1} d \zeta
$$

where $\operatorname{Re}(\alpha)>0, \beta \in \mathbb{C} \backslash \mathbb{Z},|\arg (\zeta-1)| \leq \pi$, which would yield another corrected version of Theorem 1 as follows.

THEOREM 3. With definitions and notation as in Theorem 1,

$$
\begin{aligned}
\sum_{n=0}^{\infty} \frac{(\lambda)_{n}}{(\lambda-\mu+1)_{n}} c_{n} \Phi^{*}\left(\lambda+n, \mu ; z_{1}, \ldots, z_{r}\right) f_{n}(x) t^{n} \\
=\frac{1}{2} \operatorname{csch}[(\mu-\lambda) \pi i] \frac{\Gamma(\mu)}{\Gamma(\lambda) \Gamma(\mu-\lambda)} \\
\quad \times \int_{0}^{(1+)} \zeta^{\lambda-1}(\zeta-1)^{\mu-\lambda-1} \Phi\left(z_{1} \zeta^{m_{1}}, \ldots, z_{r} \zeta^{m_{r}}\right) F\left(x, \frac{\zeta t}{\zeta-1}\right) d \zeta
\end{aligned}
$$

and

$$
\begin{aligned}
\sum_{n=0}^{\infty} \frac{(1-\mu)_{n}}{(\lambda-\mu+1)_{n}} c_{n} \Phi^{*}\left(\lambda, \mu-n ; z_{1}, \ldots, z_{r}\right) f_{n}(x) t^{n} \\
=\frac{1}{2} \operatorname{csch}[(\mu-\lambda) \pi i] \frac{\Gamma(\mu)}{\Gamma(\lambda) \Gamma(\mu-\lambda)} \\
\quad \times \int_{0}^{(1+)} \zeta^{\lambda-1}(\zeta-1)^{\mu-\lambda-1} \Phi\left(z_{1} \zeta^{m_{1}}, \ldots, z_{r} \zeta^{m_{r}}\right) F\left(x,-\frac{t}{\zeta-1}\right) d \zeta
\end{aligned}
$$

provided that $\operatorname{Re}(\lambda)>0, \mu-\lambda \in \mathbb{C} \backslash \mathbb{Z}$ and $|\arg (\zeta-1)| \leq \pi$.

Finally, in our attempt to correct Theorem 1 without using contour integrals, we are easily led to the next theorem.

THEOREM 4. With definitions and notation as in Theorem 1,

$$
\begin{aligned}
& \sum_{n=0}^{\infty} \frac{(\lambda)_{\rho n}(\mu-\lambda)_{\sigma n}}{(\mu)_{(\rho+\sigma) n}} c_{n} \Phi^{*}\left(\lambda+\rho n, \mu+(\rho+\sigma) n ; z_{1}, \ldots, z_{r}\right) f_{n}(x) t^{n} \\
& \quad=\frac{\Gamma(\mu)}{\Gamma(\lambda) \Gamma(\mu-\lambda)} \int_{0}^{1} \frac{u^{\lambda-1} \Phi\left(z_{1} u^{m_{1}}, \ldots, z_{r} u^{m_{r}}\right)}{(1-u)^{\lambda-\mu+1}} F\left(x, t u^{\rho}(1-u)^{\sigma}\right) d u,
\end{aligned}
$$

provided that $\operatorname{Re}(\mu)>\operatorname{Re}(\lambda)>0, \rho \geq 0, \sigma \geq 0$ and $\rho+\sigma>0$. 


\section{Generating functions associated with Lauricella functions}

Motivated essentially by some earlier results of Saran [7] involving the Gauss hypergeometric function ${ }_{2} F_{1}$, Mohammad $[6$, p. 262] also gave two theorems on bilinear and bilateral generating functions associated with the Appell functions $F_{2}$ and $F_{4}$ (cf. [1] and [10, pp. 22-23]), where $F_{2}=F_{A}^{(2)}$ and $F_{4}=F_{C}^{(2)}$ in terms of the aforementioned Lauricella functions $F_{A}^{(r)}$ and $F_{C}^{(r)}$ in $r$ variables, defined by (cf. [4, p. 113] and [10, p. 33])

$$
\begin{aligned}
& F_{A}^{(r)}\left[\alpha, \beta_{1}, \ldots, \beta_{r} ; \gamma_{1}, \ldots, \gamma_{r} ; z_{1}, \ldots, z_{r}\right] \\
& \quad=\sum_{k_{1}, \ldots, k_{r}=0}^{\infty} \frac{(\alpha)_{k_{1}+\cdots+k_{r}}\left(\beta_{1}\right)_{k_{1}} \cdots\left(\beta_{r}\right)_{k_{r}}}{\left(\gamma_{1}\right)_{k_{1}} \cdots\left(\gamma_{r}\right)_{k_{r}}} \frac{z_{1}^{k_{1}}}{k_{1} !} \cdots \frac{z_{r}^{k_{r}}}{k_{r} !},
\end{aligned}
$$

where $\left|z_{1}\right|+\cdots+\left|z_{r}\right|<1$, and

$$
\begin{aligned}
& F_{C}^{(r)}\left[\alpha, \beta ; \gamma_{1}, \cdots, \gamma_{r} ; z_{1}, \cdots, z_{r}\right] \\
& \quad=\sum_{k_{1}, \ldots, k_{r}=0}^{\infty} \frac{(\alpha)_{k_{1}+\cdots+k_{r}}(\beta)_{k_{1}+\cdots+k_{r}}}{\left(\gamma_{1}\right)_{k_{1}} \cdots\left(\gamma_{r}\right)_{k_{r}}} \frac{z_{1}^{k_{1}}}{k_{1} !} \cdots \frac{z_{r}^{k_{r}}}{k_{r} !},
\end{aligned}
$$

where $\left|z_{1}\right|^{1 / 2}+\cdots+\left|z_{r}\right|^{1 / 2}<1$. Indeed it is known for these multivariable hypergeometric functions that $[10, \mathrm{pp} .285-286]$

$$
\begin{aligned}
F_{A}^{(r)}\left[\alpha, \beta_{1}, \ldots, \beta_{r} ; \gamma_{1}, \ldots, \gamma_{r} ; z_{1}, \ldots, z_{r}\right] \\
\quad=\frac{1}{\Gamma(\alpha)} \int_{0}^{\infty} e^{-u} u^{\alpha-1} \prod_{j=1}^{r}\left\{F_{1}\left(\beta_{j} ; \gamma_{j} ; z_{j} u\right)\right\} d u,
\end{aligned}
$$

where $\operatorname{Re}(\alpha)>0$ and $\operatorname{Re}\left(z_{1}+\cdots+z_{r}\right)<1$, and

$$
\begin{aligned}
& F_{C}^{(r)}\left[\alpha, \beta ; \gamma_{1}, \ldots, \gamma_{r} ; z_{1}, \ldots, z_{r}\right] \\
& \quad=\frac{1}{\Gamma(\alpha) \Gamma(\beta)} \int_{0}^{\infty} \int_{0}^{\infty} e^{-u-v} u^{\alpha-1} v^{\beta-1} \prod_{j=1}^{r}\left\{F_{1}\left(-; \gamma_{j} ; z_{j} u v\right)\right\} d u d v
\end{aligned}
$$

where $\operatorname{Re}(\alpha)>0, \operatorname{Re}(\beta)>0$ and $\left|z_{1}\right|^{1 / 2}+\cdots+\left|z_{r}\right|^{1 / 2}<1$.

Making use of the integral representations (21) and (22), it is not difficult to prove Theorems 5 and 6 below.

THEOREM 5. Let the generating function $F(x, t)$ and the Lauricella function $F_{A}^{(r)}$ be given by (1) and (19), respectively. 
Then

$$
\begin{aligned}
& \frac{1}{\Gamma(\alpha)} \int_{0}^{\infty} e^{-u} u^{\alpha-1} \prod_{j=1}^{r}\left\{_{1} F_{1}\left(\beta_{j} ; \gamma_{j} ; z_{j} u\right)\right\} F(x, t u) d u \\
& \quad=\sum_{n=0}^{\infty}(\alpha)_{n} c_{n} F_{A}^{(r)}\left[\alpha+n, \beta_{1}, \ldots, \beta_{r} ; \gamma_{1}, \ldots, \gamma_{r} ; z_{1}, \ldots, z_{r}\right] f_{n}(x) t^{n},
\end{aligned}
$$

provided that each side of (23) exists.

THEOREM 6. Let the generating function $F(x, t)$ and the Lauricella function $F_{C}^{(r)}$ be given by (1) and (20), respectively.

Then

$$
\begin{gathered}
\frac{1}{\Gamma(\alpha) \Gamma(\beta)} \int_{0}^{\infty} \int_{0}^{\infty} e^{-u-v} u^{\alpha-1} v^{\beta-1} \prod_{j=1}^{r}\left\{_{0} F_{1}\left(-; \gamma_{j} ; z_{j} u v\right)\right\} F(x, t u v) d u d v \\
=\sum_{n=0}^{\infty}(\alpha)_{n}(\beta)_{n} c_{n} F_{C}^{(r)}\left[\alpha+n, \beta+n ; \gamma_{1}, \ldots, \gamma_{r} ; z_{1}, \ldots, z_{r}\right] f_{n}(x) t^{n}
\end{gathered}
$$

provided that each side of (24) exists.

In their special cases when $r=2$ and $c_{n}=1\left(n \in \mathbb{N}_{0}\right)$, Theorem 5 would correspond to the corrected version of a result of Mohammad [6, p. 262, Theorem 2], and Theorem 6 would yield another result of Mohammad [6, p. 262, Theorem 3].

Starting from numerous examples of such generating functions as $F(x, t)$ in (1) already available in the treatise by Srivastava and Manocha [11] and elsewhere (see, for example, [2, 8] and [9]), each of our results (Theorems 2 to 6 above) can indeed be applied in a manner (which is described and illustrated fairly adequately by Saran [7], Mathur [5] and Mohammad [6]) in order to derive the corresponding bilinear and bilateral generating functions for various families of special functions and polynomials in one, two and more variables. Moreover, each of our last results (Theorems 5 and 6 above) can easily be extended appropriately to hold true not only for the corresponding multiple Eulerian integrals, but also for such general classes of multivariable functions as those defined by (3) and (4). The details involved in these derivations are being left as an exercise for the interested reader.

\section{Acknowledgments}

The present investigation was initiated during the first-named author's visits to the Institute of Mathematics (Acdemia Sinica) at Taipei, National Chiao Tung University 
at Hsin-Chu, Soochow University at Taipei and Chung Yuan Christian University at Chung-Li in February 1998. This work was supported, in part, by the Natural Sciences and Engineering Research Council of Canada under Grant OGP0007353 and the National Science Council of the Republic of China under Grant NSC-882115-M001-001.

\section{References}

[1] P. Appell, "Sur les séries hypergéométriques de deux variables, et sur des équations différentielles linéaires aux dérivées partielles", C. R. Acad. Sci. Paris 90 (1880) 296-298.

[2] M.-P. Chen and H. M. Srivastava, "Some families of bilinear and bilateral generating functions", Comput. Math. Appl. 28 (9) (1994) 1-7.

[3] A. Erdélyi, W. Magnus, F. Oberhettinger and F. G. Tricomi, Higher transcendental functions, Vol. I (McGraw-Hili, New York, 1953).

[4] G. Lauricella, "Sulle funzioni ipergeometriche a più variabili", Rend. Circ. Mat. Palermo 7 (1893) 111-158.

[5] B. L. Mathur, "On some results involving Lauricella functions", Bull. Calcutta Math. Soc. 70 (1978) 221-227.

[6] Ch. W. Mohammad, "Bilinear and bilateral generating functions of generalized polynomials", $J$. Austral. Math. Soc. Ser. B 39 (1997) 257-270.

[7] S. Saran, "Theorems on bilinear generating functions", Indian J. Pure Appl. Math. 3 (1972) 12-20.

[8] H. M. Srivastava, "Orthogonality relations and generating functions for the generalized Bessel polynomials", Appl. Math. Comput. 61 (1994) 99-134.

[9] H. M. Srivastava and L. C. Gupta, "Some families of generating functions for the Jacobi polynomials", Comput. Math. Appl. 29 (4) (1995) 29-35.

[10] H. M. Srivastava and P. W. Karlsson, Multiple Gaussian hypergeometric series (Ellis Horwood Ltd., Chichester, 1985).

[11] H. M. Srivastava and H. L. Manocha, A treatise on generating functions (Ellis Horwood Ltd., Chichester, 1984).

[12] E. T. Whittaker and G. N. Watson, A course of modern analysis: An introduction to the general theory of infinite processes and of analytic functions; with an account of the principal transcendental functions, Reprint of the fourth (1927) edition (Cambridge University Press, Cambridge, 1996). 